\title{
Observation of dipolar ferromagnetism by TEM techniques
}

\author{
M. Beleggia, ${ }^{*}$ S. Tandon, ** Y. Zhu, ${ }^{*}$ and M. De Graef**
}

* Center for Functional Nanomaterials, Brookhaven National Laboratory, Upton, NY 11973

*** Dept. of Materials Science and Engineering, Carnegie Mellon University, Pittsburgh, PA 15213

Magnetostatic interactions play a central role in determining the magnetic response of an array of patterned magnetic elements or magnetized nanoparticles of given shape. The Fourier space approach recently introduced for the analytical computation of the demagnetizing tensor, field and energy for particles of arbitrary shape [1], has been recently extended to cover interactions between elements [2]. The main achievement has been the definition of a generalized dipole-dipole interaction for cylindrical objects, which takes into account the influence of shape anisotropy without resorting to any kind of approximations [3]. Once the interaction energy is available, it becomes possible to evaluate the minimum energy state of the system for a given set of external parameters (such as applied field, aspect ratio, distance between elements). It turns out that, below a critical combination of aspect ratios and distances, the system undergoes a phase transition, changing abruptly from a closure domain state, with zero net magnetization, to a dipolar ferromagnetic state with a net magnetization which depends on the interaction strength between the elements.

In order to observe this phenomenon by TEM, it is necessary to estimate the feasibility of the experiment by a series of simulations. Figure 1 shows the electron optical phase shifts for the system of three weakly (a-c) and strongly (d) interacting disks. The elements have a radius of $50 \mathrm{~nm}$, a thickness of $5 \mathrm{~nm}$ (aspect ratio 1/20), and are uniformly magnetized at 2 T. By comparing Fig.1 (c) and (d), it can be seen that the fingerprint of the transition is the presence of fringing fields around the elements, revealed by the cosine fringes which resemble a dipole-like field in (d). When the system is in the closure-domain state, as in (a-c), no fringing field can be observed on a large scale. Figure 2 shows a set of Fresnel images, calculated for the same configurations as in Fig.1. As the Fresnel technique is scarcely sensitive to fringing fields, their effect is not evident in the images. However, by following the bright and dark contrast features in each image, a change in magnetization can be deduced, also when the electrostatic phase shift proportional to the thickness is taken into account.

An alternative, more indirect, opportunity to observe the transition is granted by hysteresis loops. Figure 3 shows calculated hysteresis curves for non-interacting (a), weakly interacting (b) and strongly interacting (c) disks. The applied field is in-plane, along one of the triangular symmetry axis $\left(0^{\circ}, 60^{\circ}\right.$ or $120^{\circ}$ ). The transition is revealed by a remanent magnetization in zero field (c), contrary to the behavior of the weak interaction (b). Dipolar ferromagnetism can, therefore, be successfully investigated by TEM, where at least three different techniques, phase retrieval, phase contrast, and insitu hysteresis experiments, are available for detecting the phase transition [4].

\section{References}

[1] M. Beleggia and M. De Graef, J. Magn. Magn. Mater. 263, L1 (2003).

[2] M. Beleggia et al., J. Magn. Magn. Mater. (2004) in press.

[3] M. Beleggia, IEEE Trans. Mag. (2004) in press.

[4] This work was supported by U.S. Department of Energy, Basic Energy Science, under contract No. DE-AC02-98CH10886. 

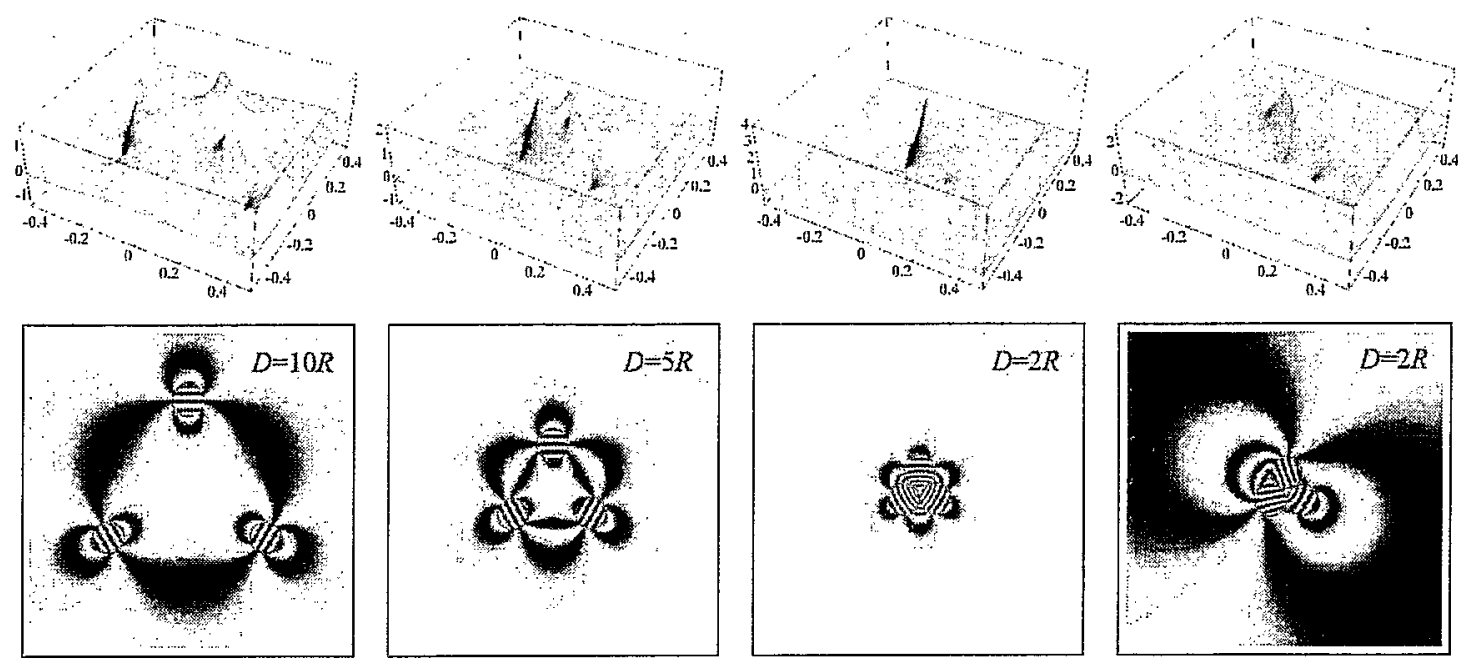

FIG. 1. Phase shift for the system of three disks displayed as surface plots (top) and $8 \times$ amplified holographic cosine maps (bottom). The distance between disks decreases from left to right. The image size is $1 \mu \mathrm{m}^{2}$, and the phase shift is measured in radians.
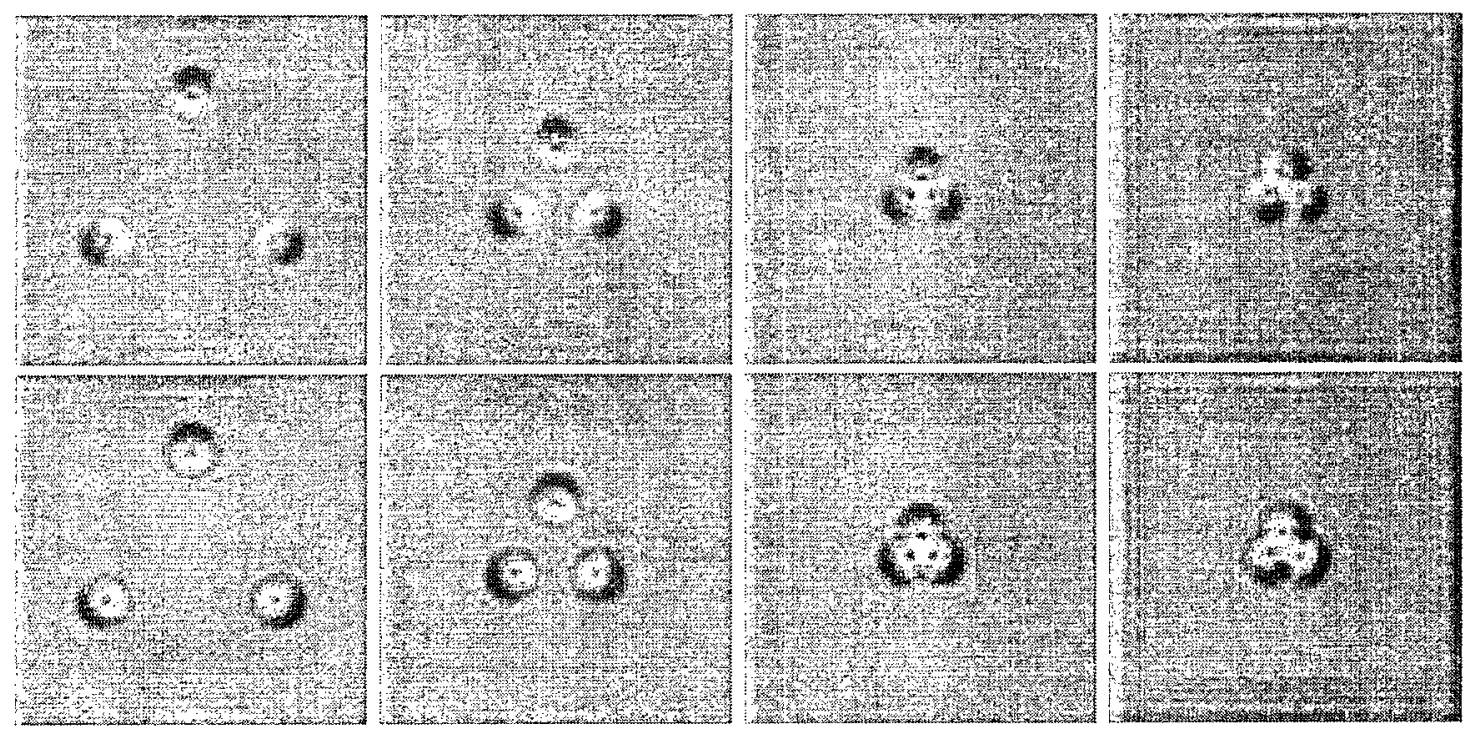

FIG. 2. Out of focus images ( $1 \mathrm{~mm}$ defocus) for the three disk system with relative distances as in Figure 1. Top row: magnetic phase only; bottom row: magnetic and electrostatic phase shifts.
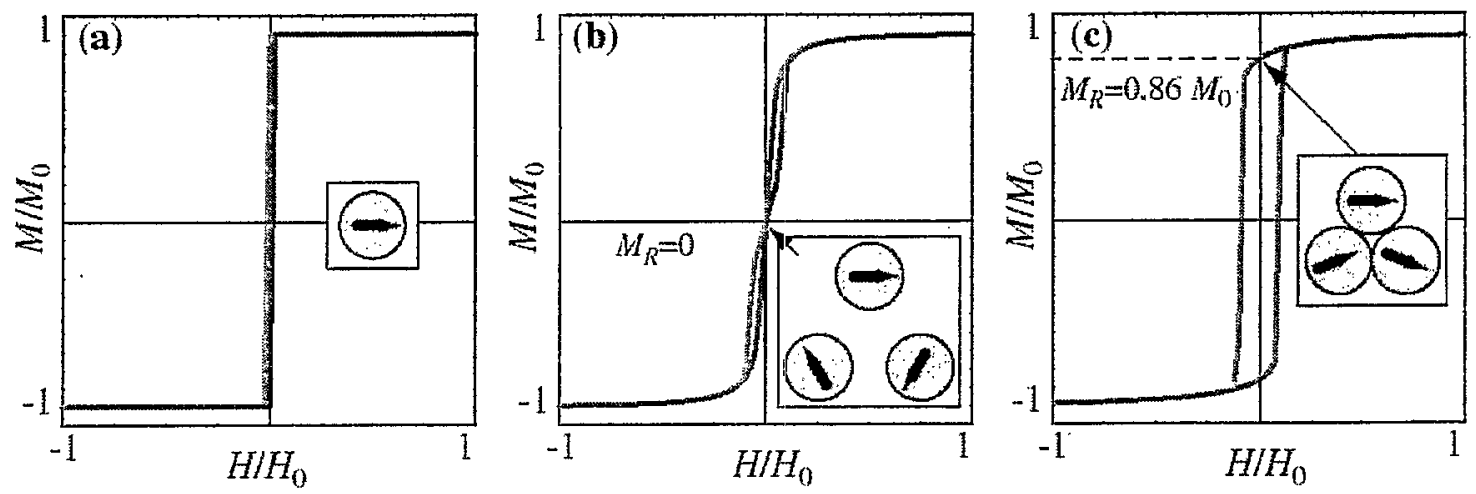

FIG. 3. Hysteresis loops for non-interacting (a, isolated disks), weakly interacting (b, above critical distance) and strongly interacting (c, below critical distance) disks under an in-plane $\left(0^{\circ}\right)$ applied field. 